\title{
Stage III Adrenal Cortex Carcinoma AJCC v7
}

National Cancer Institute

\section{Source}

National Cancer Institute. Stage III Adrenal Cortex Carcinoma A/CC v7. NCI Thesaurus. Code C9216.

Stage III includes: (T1, N1, M0); (T2, N1, M0); (T3, N0, M0). T1: T umor $5 \mathrm{~cm}$ or less in greatest dimension, no extra-adrenal invasion. T2: T umor greater than $5 \mathrm{~cm}$, no extraadrenal invasion. T3: T umor of any size with local invasion, but not invading adjacent organs. N1: Metastasis in regional lymph node(s). N0: No regional lymph node metastasis. M0: No distant metastasis. (AJCC 7th ed.) 